\title{
Benno Gammerl, Anders Fühlen: Schwules und lesbisches Leben in der Bundesrepublik. Eine Emotionsgeschichte.
}

Sébastien Tremblay

\section{(2) OpenEdition}

Electronic version

URL: https://journals.openedition.org/ifha/11552

DOI: 10.4000/ifha. 11552

ISSN: 2198-8943

Publisher

IFRA - Institut franco-allemand (sciences historiques et sociales)

Electronic reference

Sébastien Tremblay, "Benno Gammerl, Anders Fühlen: Schwules und lesbisches Leben in der Bundesrepublik. Eine Emotionsgeschichte.", Revue de l'IFHA [Online], Date of review, Online since 07 December 2021, connection on 13 December 2021. URL: http://journals.openedition.org/ifha/11552 ; DOI: https://doi.org/10.4000/ifha. 11552

This text was automatically generated on 13 December 2021.

(C)IFHA 


\section{Benno Gammerl, Anders Fühlen:} Schwules und lesbisches Leben in der Bundesrepublik. Eine Emotionsgeschichte.

Sébastien Tremblay

\section{REFERENCES}

Benno Gammerl, Anders Fühlen: Schwules und lesbisches Leben in der Bundesrepublik. Eine Emotionsgeschichte, Munich: Hanser, 2021, 416p., 25,00€. 
En 2016, Jennifer Evans rappelait dans un article phare dans les pages de German History que l'histoire queer ne consiste pas seulement à diversifier une histoire LGBT, mais aussi à réfléchir méthodologiquement sur l'écriture de l'histoire gaie et lesbienne, soulignant la fluidité des catégories d'analyses tel le genre et une déconstruction des binarités sexuelles. Ayant écrit l'une des premières manifestations d'un tel projet dans la littérature queer publiée en langue allemande, Benno Gammerl, professeur d'histoire du genre et de la sexualité à l'Institut universitaire européen de Florence, offre ici une excellente chronologie de l'histoire gaie et lesbienne en RFA et un ouvrage charnière pour l'historiographie de l'histoire allemande contemporaine. Il fait écho à un courant

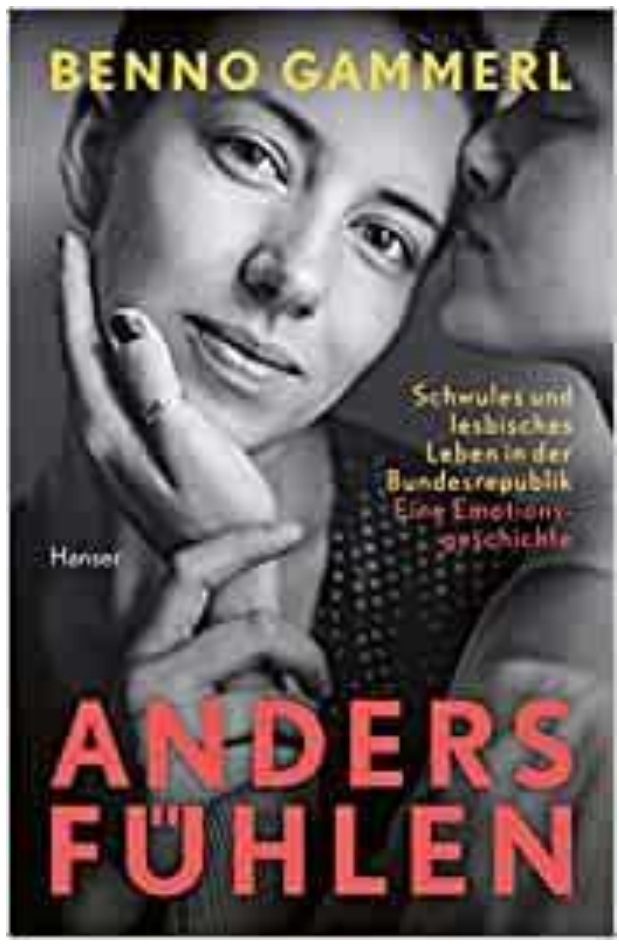
similaire dans le monde des Germanistes anglo-saxons revisitant des moments importants de la seconde partie du XXe siècle en RFA, essayant de mettre en évidence les contestations, les conflits et les différentes facettes de l'histoire LGBT. Cet ouvrage est également louable pour son inclusion d'une histoire orale gaie et lesbienne. Ainsi trente-deux entrevues réalisées avec des hommes et des femmes issu-es de la communauté gaie et lesbienne et né-es entre 1935 et 1970 sont généreusement analysées et forment le cœur de l'argumentaire. Gammerl joint à ces témoignages un corpus impressionnant de textes et d'articles sur les moments clés de l'histoire homosexuelle et une revue de presse de différents périodiques LGBT de la même époque (p.28-31).

L'auteur s'appuie sur l'histoire des émotions pour analyser l'histoire LGBT ouestallemande sous un nouvel angle (p.25). Plus concrètement, il cadre son étude dans une nouvelle périodisation basée sur les émotions de ses interlocuteur-trices, nuançant une historiographie gaie et lesbienne qui relie usuellement un passé de persécution à un présent soi-disant progressiste (p.344). Gammerl propose ainsi trois espaces temporels différents où ces hommes et femmes se cachent, se dévoilent au grand jour, sont en colère, tristes, amoureux-ses ou luttent pour l'avancement de leurs droits. À travers son examen de l'aspect toujours changeant de leurs peurs et de leurs aspirations politiques (p.339-341), l'auteur démontre comment l'écriture d'une histoire LGBT doit délaisser la commémoration nostalgique d'un radicalisme soi-disant perdu des années soixante-dix (p.155) et une tendance à considérer les années quatre-vingt comme une période de stagnation (p.260-262).

L'ouvrage est séparé en trois parties distinctes: Ausweichen (l'évasion), Aufbrechen! (éclater au grand jour!) Ankommen? (Arriver?), entrecoupées et introduites par de long extraits extirpés de deux entrevues: avec Frau Schmidt et Herr Meyer. La première partie s'attarde au mouvement homophile des années d'après-guerre et à la valse quotidienne entre un silence imposé et une vie homosexuelle plus ou moins secrète. La 
deuxième se concentre sur les mouvances émancipatrices des révolutions sexuelles et de la création des associations homosexuelles faisant suite à la décriminalisation de l'homosexualité masculine et aux changements de mœurs subséquents la période Adenauer (p.46). Ces deux parties démontrent bien en quoi l'histoire gaie et lesbienne remet en question une historiographie présentant les années soixante comme une période de libéralisation, ce moment n'arrivant qu'à la fin de cette décennie pour les homosexuel-les. Gammerl réussit malgré tout à ne pas présenter les années cinquante et soixante comme une antichambre aux tendances radicales des années soixante-dix, soulignant le caractère pluriel des associations dites homophiles ou homosexuelles. La troisième partie de l'ouvrage et son épilogue remettent ensuite en question une tendance historiographique présentant les années quatre-vingt comme une simple histoire d'institutionnalisation et d'assimilation. En se concentrant encore une fois sur la peur mais aussi le double-lien entre la honte et la fierté, Gammerl inclut les luttes pour le mariage pour tous et toutes (p.345) et les luttes homosexuelles liées au sida (p. 273) dans une histoire multidimensionnelle des luttes LGBT. Débouchant éventuellement sur l'iconoclasme queer de la fin du XXe siècle, Gammerl dresse un tableau des contradictions et des similitudes d'un demi-siècle de politique gaie et lesbienne sans simplement opposer radicalisme et embourgeoisement.

Le livre fait ainsi fi des carcans binaires d'analyse. Déjà dans l'introduction (p.21), l'auteur mentionne qu'entre deux pôles, une dissimulation honteuse et un affichage complet de la sexualité, d'innombrables possibilités s'articulent plus ou moins clairement: privé ou public (p.79), gai ou lesbienne, politique libérale ou radicale, liberté urbaine ou isolement rural (p.98), honte ou fierté, aspirations négatives ou positives vers le passé, émancipation ou assimilation. Gammerl écarte la pensée binaire, préférant inclure les témoignages collectés sur des spectres conceptuels plus souples (p.139). Anders Fühlen est donc aussi une contribution importante à la façon dont l'historiographie queer conçoit l'espace (p.24). Cela ne veut pas dire que l'auteur n'identifie pas des différences claires entre l'histoire gaie et lesbienne (p.181). En effet, trop souvent l'histoire lesbienne est présentée comme un simple complément à une écriture de l'histoire gaie. S'appuyant sur ses entrevues, Gammerl souligne l'importance du genre. Il mentionne, par exemple, à quel point l'évasion n'était pas la même pour un homme gai ou une femme lesbienne coincé-es dans un mariage hétérosexuel dans les années soixante en raison de l'accès à l'espace public ou professionnel (p.83).

L'histoire et l'écriture de l'histoire deviennent aussi un espace de réflexion tout au long de l'ouvrage. Gammerl le démontre très bien en réfléchissant sur le lien l'unissant à ses interlocuteur.trices. Un cas est particulièrement frappant, son entretien avec Herr Schumann. Provoqué et irrité par la manière qu'a Schumann de le forcer dans une communauté émotionnelle partagée au cours du processus de recherche, l'auteur offre plusieurs apartés sur sa position dans cet espace historiographique (p.114-118).

Cette importante contribution n'est pourtant pas sans bémol. Si la nouvelle chronologie offerte par l'auteur convainc, une histoire de la RFA post-unification ne s'attardant pas à la RDA semble incomplète. Il ne s'agit pas ici d'une critique du focus ouest-allemand, mais plutôt d'une certaine irritation quant à un manque de contributions de milliers de personnes arrivant en RFA suite à l'unification des deux pays. De même, on aurait souhaité de plus amples détails sur la manière dont la séparation des deux Allemagnes a pu affecter les gens partageant leurs histoires. Malgré cela, le livre est non seulement un excellent ouvrage d'histoire gaie et lesbienne, mais peut-être finalement le livre qui 
donnera ses lettres de noblesse à l'histoire orale et à l'histoire queer dans le monde académique germanophone.

INDEX

Chronological index: Époque contemporaine

Subjects: Histoire de la culture, Histoire des femmes et du genre, Histoire sociale

Mots-clés: Histoire de la culture

\section{AUTHOR}

\section{SÉBASTIEN TREMBLAY}

Freie Universität Berlin, Friedrich-Meinecke-Institut, Arbeitsbereich Globalgeschichte, Goldsmiths University of London, Centre for Queer History 